\title{
Need for public debate about fertility treatments
}

\section{Manipulations of the mitochondrial germ line must be openly debated and followed up.}

Sir - Barritt et al. ${ }^{1}$ have reported the birth of several 'transmitochondrial babies' following ooplasmic transplantation at in-vitro fertilization (IVF) centres around the world. In this technique, about 10\% of the ooplasm is transferred from a healthy donor egg into a compromised patient's egg ${ }^{2}$. Because normal human oocytes contain between 200,000 and 5 million mitochondria, the transferred ooplasm probably contains about 20,000-500,000 mitochondria and mitochondrial (mt) DNAs, together with other cytoplasmic components.

Ooplasmic transplantation involves the mixing of mtDNAs in the cytoplasmic germ line. Because this may produce durable and unknown genetic effects that transcend single generations, this new technology must be scrutinized both publicly and scientifically. The social, medical and legal questions about the birth of children who bear the DNA of three parents - mtDNA from the biological mother and the ooplasm donor, and nuclear DNA from both biological parents - cannot be fully anticipated without public discussion and timely scientific publication of results. Cohen and his colleagues are to be commended for their open reporting of rare cases of aneuploidy and other complications associated with ooplasmic transplantation $^{3}$.

We agree with Parens and Juengst ${ }^{4}$ that increased public supervision is needed. Along these lines, the US Food and Drug Administration (FDA) issued a letter on 6 July 2001 (www.fda.gov/cber/ltr/ cytotrans070601.htm) to investigators using ooplasmic transplantation and other forms of gene transfer in human gametes. Investigators using these technologies are now required to submit an 'investigational new drug' (IND) application to the FDA, outlining the research methods and objectives of the clinical study.

We wish to make two additional recommendations. First, we suggest that the National Institutes of Health (NIH) expands its Recombinant DNA Advisory Committee, or creates a new regulatory body, to encompass all ooplasm and nuclear-transfer technologies, to allow informed regulation and public reporting. Until a more solid public and scientific database is established, the number of licences issued should be limited to a handful of highly qualified, excellent IVF facilities. Each of these should be required to follow certain protocol-driven data collection and publication procedures.
Data collection would include haplotype analysis of donor and recipient mtDNAs, screening for the most common pathogenic mtDNA mutations, documentation of gestational complications and maternal-fetal immunological and metabolic parameters, and careful neurodevelopmental testing of the infants and toddlers. The NIH regulatory body should include: people with expertise in a wide range of IVF technologies, including ooplasmic transplantation; specialists in medical ethics, nursing, law, mitochondrial cell biology, mitochondrial medicine and genetics; and informed members of the general public.

Our second recommendation is that the US Congress set aside a budget for a request for applications, issued by the $\mathrm{NIH}$, to solicit proposals to study animal models of the ooplasmic transfer technology. Although significant biological differences make direct comparison of the details of reproductive cell biology between species difficult, these studies would provide the basic data needed to understand the pros and cons of ooplasmic transfer technology more fully ${ }^{5}$. It is only with carefully regulated progress, timely and systematic publication, public supervision and public debate that we can maintain public trust in science, while moving forward in the genomic and postgenomic ages of medicine that lie ahead.

Robert K. Naviaux ${ }^{*}$, Keshav K. Singh $\dagger$

${ }^{*}$ The Mitochondrial and Metabolic Disease Center, University of California, San Diego, 214 Dickinson Street, CTF, C-103, San Diego, California 921038467, USA

†Johns Hopkins Oncology Center, Bunting-Blaustein Cancer Research Building, 1650 Orleans Street, Room 1-143, Baltimore, Maryland 21231-1000, USA

1. Barritt, J. A., Brenner, C. A., Malter, H. E. \& Cohen, J. J. Hum. Reprod. 16, 513 (2001).

2. Cohen, J., Scott, R., Schimmel, T., Levron, J. \& Willadsen, S. Lancet 350, 186 (1997).

3. Barritt, J. A., Brenner, C. A., Willadsen, S. \& Cohen, J. J. Hum. Reprod. 15, 207 (2000).

4. Parens, E. \& Juengst, E. Science 292, 397 (2001).

5. Thorburn, D. R., Dahl, H.-H. M. \& Singh, K. K. Mitochondrion 1, 123 (2001).

\section{Risk that websites could break code of anonymity}

Sir-Anonymous peer review is the fundamental process by which the scientific community evaluates grant proposals. One way in which the Internet has changed the review process is that granting agencies now allow, and some encourage, the construction of websites by authors to supplement proposals. Although these sites provide useful methodological and other information to reviewers, they threaten reviewer anonymity.

Server software can log the unique IP address of each computer that visits a particular website. These IP addresses can identify the institution and, in some cases, the name of the visitor. This is especially problematic when reviewers are the only visitors to a site created specifically for the proposal and hosted by the author, who has disclosed the URL only in the proposal. Reviewers who are aware of the risk of exposing their identity in this way may avoid websites associated with proposals, effectively rendering their review incomplete.

We contacted several funding agencies (NSF, USDA, NERC and the Wellcome Trust), and none has a policy regarding the use of websites in conjunction with grant proposals, nor do they have mechanisms to protect the anonymity of reviewers visiting proposal websites.

In the short term, we suggest that reviewers visit such sites using a dial-up Internet service provider (for example, America Online and NetZero) that will not provide any information about the reviewer's identity. As a long-term solution, granting agencies should consider hosting such websites rather than allowing submitting authors to host them at their home institution.

Whereas this Correspondence is primarily concerned with the grant review process, the same problem applies to articles submitted to journals for publication, if supplementary material essential to evaluate the article is supplied for peer-reviewers at an author's own website.

David L. Reed, Brett Moyer, Dale Clayton Department of Biology, University of Utah, 257 South 1400 East, Room 201, Salt Lake City, Utah 84112, USA

Nature has always required supplementary information to be submitted to our editorial offices for peer-review and editorial evaluation. We do not allow such material to be hosted on an author's own site. See guide to authors at http:// www.nature.com/nature/submit/gta/ index.html\#4.10 — Editor, Nature 